\title{
Chromosomal instability in Streptomyces avermitilis: major deletion in the central region and stable circularized chromosome
}

\author{
Wei Chen, Fei He, Xiaojuan Zhang, Zhi Chen, Ying Wen, Jilun Li
}

\begin{abstract}
Background: The chromosome of Streptomyces has been shown to be unstable, frequently undergoing gross chromosomal rearrangements. However, the mechanisms underlying this phenomenon remain unclear, with previous studies focused on two chromosomal ends as targets for rearrangements. Here we investigated chromosomal instability of Streptomyces avermitilis, an important producer of avermectins, and characterized four gross chromosomal rearrangement events, including a major deletion in the central region. The present findings provide a valuable contribution to the mechanistic study of genetic instability in Streptomyces.

Results: Thirty randomly-selected "bald" mutants derived from the wild-type strain all contained gross chromosomal rearrangements of various types. One of the bald mutants, SA1-8, had the same linear chromosomal structure as the high avermectin-producing mutant 76-9. Chromosomes of both strains displayed at least three independent chromosomal rearrangements, including chromosomal arm replacement to form new 88-kb terminal inverted repeats (TIRs), and two major deletions. One of the deletions eliminated the 36-kb central region of the chromosome, but surprisingly did not affect viability of the cells. The other deletion (74-kb) was internal to the right chromosomal arm. The chromosome of another bald mutant, SA1-6, was circularized with deletions at both ends. No obvious homology was found in all fusion sequences. Generational stability analysis showed that the chromosomal structure of SA1-8 and SA1-6 was stable.

Conclusions: Various chromosomal rearrangements, including chromosomal arm replacement, interstitial deletions and chromosomal circularization, occurred in S. avermitilis by non-homologous recombination. The finding of an inner deletion involving in the central region of S. avermitilis chromosome suggests that the entire Streptomyces chromosome may be the target for rearrangements, which are not limited, as previously reported, to the two chromosomal ends.
\end{abstract}

\section{Background}

Streptomyces are a genus of Gram-positive, filamentous soil bacteria, which display complex morphological differentiation and produce a broad range of bioactive secondary metabolites such as antibiotics, immunosuppressants and cholesterol-lowering agents. These bacteria thus provide an important natural source of commercial products for the pharmaceutical and agricultural industries [1]. The Streptomyces genome consists of an 8- to 9-Mb linear chromosome, characterized by terminal inverted repeats (TIRs) and a protein covalently attached to 5' end [2-4].

\footnotetext{
* Correspondence: wen@cau.edu.cn

State Key Laboratories for Agro-biotechnology, College of Biological Sciences, China Agricultural University, Beijing 100193, PR China
}

This chromosome is inherently unstable, and frequently undergoes gross chromosomal rearrangements spontaneously as well as under various mutagenic treatments $[5,6]$, particularly in terminal regions where almost no essential genes reside. Gross chromosomal rearrangements include deletion, amplification, arm replacement, and circularization [7-16]. This chromosomal instability leads to genetic instability, which is ubiquitous among Streptomyces, and affects nearly all life functions, e.g., differentiation, secondary metabolism, and response to environmental changes [5]. The chromosomal instability is not attributable to the linear chromosomal structure, since some mutants with circular chromosomes display even higher frequency of genetic instability $[7,17,18]$. 
Theoretically, gross chromosomal rearrangements can arise through both homologous recombination and nonhomologous recombination pathways. However, the mechanisms underlying these types of rearrangement in Streptomyces are poorly understood.

Streptomyces avermitilis produces avermectins (macrocyclic lactone derivatives with potent anthelmintic properties) which are widely used in agriculture, veterinary medicine, and human medicine $[4,19]$. Sequencing of the 9.02-Mb genome of $S$. avermitilis has been completed [4]. Comparative analysis with S. coelicolor A3(2) revealed that $S$. avermitilis has a highly conserved 6.5 $\mathrm{Mb}$ "core" internal region and two variable "auxiliary" telomeric regions: a $2.0 \mathrm{Mb}$ left arm and a $0.5 \mathrm{Mb}$ right arm. The TIRs are solely located within the first 174 nucleotides at both ends of the chromosome [20]. Genetic instability had been well studied in several other Streptomyces species [10-16,21-24]. S. avermitilis, although not yet systematically investigated in this regard, is clearly subjected to genetic instability as well, since it frequently generates "white" or "bald" mutants showing reduction or complete loss of avermectin productivity. Such genetic instability is a significant problem for the commercial use of $S$. avermitilis in the fermentation industry as well as basic research, and therefore a better understanding of the mechanisms involved is needed.

In the present work, we examined the genetic instability of $S$. avermitilis using a combined approach of pulsed-field gel electrophoresis (PFGE), Southern hybridization, PCR, and DNA sequencing. The chromosomal structures of two bald mutants, SA1-6 and SA1-8, derived from spontaneous chromosomal rearrangement of the wild-type strain, were characterized in detail. Major deletion in the central region of the Streptomyces chromosome was observed for the first time in SA1-8, and stable circularized chromosome was observed in SA1-6. Analysis of the fusion sequences showed that non-homologous recombination was involved in the chromosomal rearrangements, including arm replacement, deletions and circularization. Lastly, the chromosome of SA1-6 and SA1-8 remained stable after ten passages, whereas other mutants such as SA1-7 and SA3-1 underwent further chromosomal rearrangements.

\section{Results}

Chromosomal instability in S. avermitilis

After serial transfer (more than 6 passages) on solid YMS medium, spores of $S$. avermitilis were harvested and suspended in distilled water. The spore suspension was re-plated on solid YMS to observe the phenomenon of morphological instability. Normal gray colonies appeared together with "white" mutants (i.e., defective in the ability to form mature spores) and bald mutants in the progeny. The mutants arose with a high frequency of $2.4 \%$ from the wild-type strain, and an even higher frequency of $8.3 \%$ from 76-9, a high avermectin-producing mutant. Thirty bald mutants from the wild-type strain and 30 bald mutants from 76-9 were randomly isolated, solely on the basis of their stable aerial mycelia-defective phenotype. Flask fermentation experiments and subsequent HPLC analysis demonstrated that all of these bald mutants lost the ability to produce avermectins (data not shown).

To test whether chromosomes of the bald mutants were altered similarly to those in other Streptomyces species as reported previously [5], we conducted PFGE analysis of chromosomal structure. Through optimal adjustment of pulse time, 25 AseI-fragments of S. avermitilis ATCC31267 (Fig. 1A) were successfully separated (except for 5-kb fragment $\mathrm{Y}$ ) and varied in size from 57$\mathrm{kb}$ to $1422-\mathrm{kb}$ (Fig. $1 \mathrm{~B}$ and $1 \mathrm{C}$ ). Fragments D and W correspond to the right and left ends of the chromosome, respectively, which covalently bind terminal proteins. In comparison to AseI patterns of wild-type chromosome, all the bald mutants derived from wildtype (designated SA) displayed chromosomal rearrangements. Some of the mutants shared similar PFGE profile representatively shown in Fig. $1 B$ and $1 C$, although the chromosomal structures among these mutants might be different. Fragments AseI-W (63-kb) and A (1422-kb) on the left chromosomal arm were involved in nearly all deletion events, most of which extended to fragment $\mathrm{U}$ $(85-\mathrm{kb})$. Considering that the overlapping band $\mathrm{D} / \mathrm{E}$ became fainter and thinner, it is most likely that the right terminal fragment $\mathrm{D}$ was missing, although the possibility that centrally located fragment E could also be missing can not be excluded. Meanwhile, some new AseI bands appeared in the SA mutants. In contrast, the spontaneous bald mutants derived from 76-9 showed no apparent chromosomal rearrangements in comparison to the AseI pattern of 76-9 (Additional file 1: Supplementary Fig. S1).

Among the rearrangement types of SA mutants, the AseI profile of SA1-6 showed no novel bands apart from the deleted fragments (Fig. $1 \mathrm{~B}$ and $1 \mathrm{C}$ ). On the other hand, the AseI profile of SA1-8 revealed two new fragments, and was quite similar to that of 76-9 (Fig. 1D), suggesting that SA1-8 and 76-9 may share the same chromosomal structure. Therefore, SA1-6 and SA1-8 were selected for further study of chromosomal architecture.

Both the linear chromosome and plasmid maintain a circular conformation in vivo because of the interaction of two terminal proteins. When intact DNA samples are treated with Proteinase K (PK), the covalently bound terminal proteins are removed and the DNA acquires a linear conformation. Whereas the intact DNA in the 


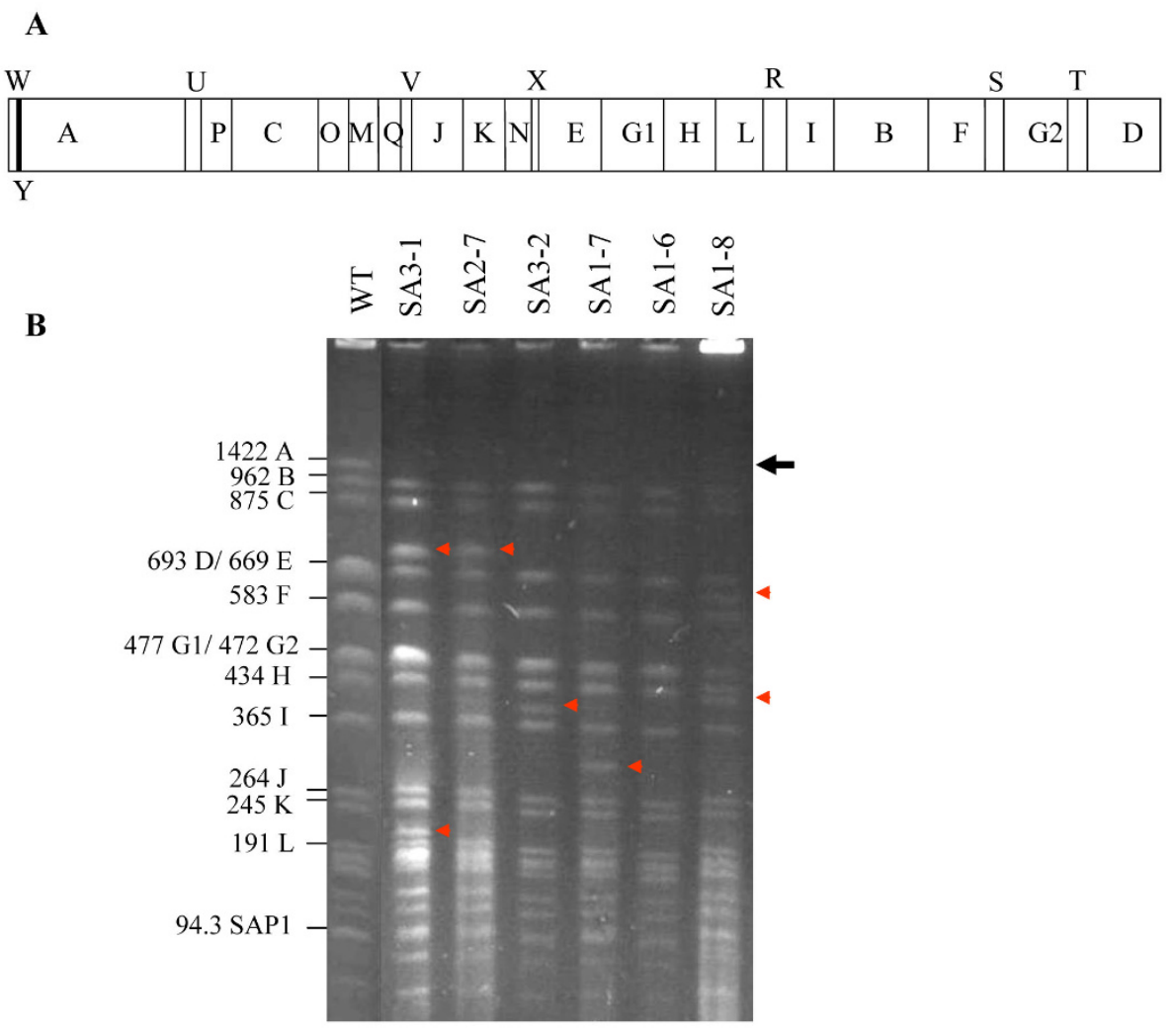

C

D

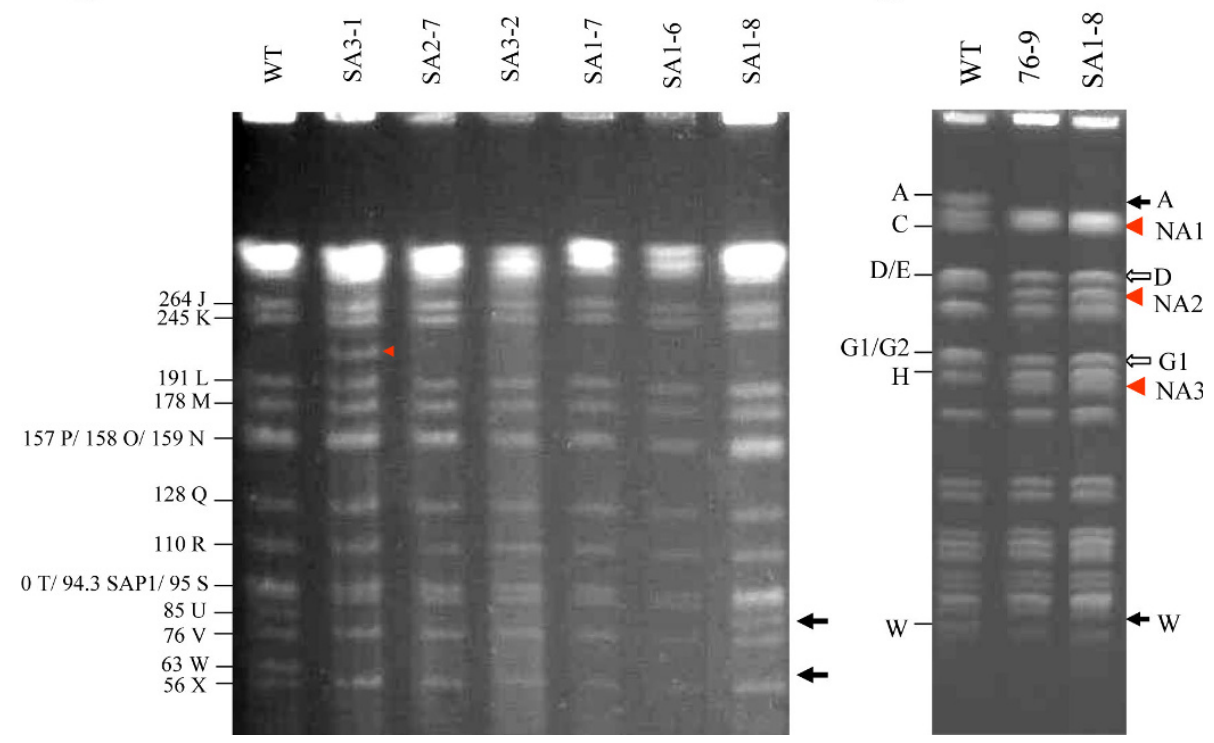

Figure 1 Gross chromosomal rearrangements in spontaneous bald mutants from S. avermitilis wild-type (WT) strain ATCC31267. (A) Asel restriction map of wild-type chromosome. (B and C) Asel restriction patterns of genomic DNA of bald mutants (SA). (D) Similar Asel profiles of 76-9 and SA1-8. PFGE conditions for separating large fragments were: (B and D) 1.2\% agarose, $4.5 \mathrm{~V} / \mathrm{cm}, 20-130 \mathrm{~s}$ pulses, $36 \mathrm{~h} ; 4.5 \mathrm{~V} / \mathrm{cm}, 60-90$ s pulses, $2 \mathrm{~h} ; 4.5 \mathrm{~V} / \mathrm{cm}, 5-10 \mathrm{~s}$ pulses, $8 \mathrm{~h}$; conditions for separating small fragments were: (C) $1.5 \%$ agarose, $6 \mathrm{~V} / \mathrm{cm}, 5-10 \mathrm{~s}$ pulses, $24 \mathrm{~h}$.

Fragments $D$ and $E$ overlapped because of their extremely similar migration; overlap was also found for fragments G1/G2, O/P/N, and S/T. SAP1: 94.3-kb linear plasmid. Solid arrows: missing fragments; Open arrows: potential missing fragments; Triangles: new bands. 
SDS-treated sample is trapped in the slot, since just noncovalently bound proteins are removed and the linear DAN keeps a circular form [3]. It has been reported that the wild-type strain ATCC31267 has a linear chromosome and a linear plasmid SAP1 of 94.3-kb [4]. When intact DNA samples of SA1-8, SA1-6 and the wild-type strain were electrophoresed under conditions allowing migration of only DNA linear structures, SA1-8 showed no detectable difference in banding pattern as compared to the wild-type strain, whereas only one plasmid band was detected in PK-treated DNA sample of SA1-6 (Fig. 2A), suggesting that the SA1-8 chromosome remained linear, whereas SA1- 6 possessed a circular chromosome.

\section{Chromosomal arm replacement and internal deletions in SA1-8 chromosome}

In comparison to the AseI profile of wild-type, fragments $\mathrm{W}$ and $\mathrm{A}$ on the left chromosomal arm of SA1-8 were missing, and there were two novel fragments, which we termed NA2 and NA3 (Fig. 1D). To test whether the deletion of the $\mathrm{W}$ fragment included the left chromosomal terminus, we used probe W (754-1653 nt, relative to left first nucleotide of the chromosome defined as $1 \mathrm{nt}$ ) located on the left terminus, to hybridize onto the PstI pattern of genomic DNA. The wild-type strain showed a predicted $1.6-\mathrm{kb}$ restriction fragment, whereas SA1-8 showed no apparent hybridization signal (Additional file 1: Supplementary Fig. S2A), indicating that the left terminus was deleted. On the other hand, the right extremity was still conserved, since hybridization with probe Dr (196-bp away from the last nucleotide) showed that the terminal $4.7-\mathrm{kb} B a m \mathrm{HI}$ fragment was present in both wild-type and SA1-8 (Additional file 1: Supplementary Fig. S2B).

Although SA1-8 lost the ability to produce avermecetins, the avermectin biosynthetic gene cluster, located within AseI-A, could be specifically amplified by PCR (data not shown), indicating that fragment $\mathrm{A}$ was not deleted completely. To determine the remnant of fragment A, probe aveC $(1,168,000-1,169,000 \mathrm{nt})$ in the ave gene cluster was amplified and labeled. Hybridization with this probe, surprisingly, revealed a new band (termed NA1) overlapping with fragment C (875-kb) (Fig. 1D and 3A). Fragment NA1 was also detected by the right terminal probe Dr, which hybridized with fragment $\mathrm{D}$ in wild-type (Fig. 3A). These results suggest that the right end replaced the left end and joined the undeleted part of AseI-A to form the novel left terminal fragment NA1.

Probe Dr hybridized simultaneously with fragments NA1 and NA2 in SA1-8, and with fragment D in wildtype, suggesting that NA2 was derived from fragment D (Fig. 3A). Hybridization with probe D600 confirmed the loss of 693-kb AseI-D, and formation of new $\sim 600-\mathrm{kb}$ NA2 in SA1-8 (Fig. 3A). When proteinase treatment was omitted, neither NA2 in SA1-8 nor AseI-W in wildtype entered the PFGE gel (Fig. 2B). Slowing of fragment $\mathrm{D}$ in wild-type and of NA1 in SA1-8 could not be observed since they overlapped with fragments $\mathrm{E}$ and $\mathrm{C}$, respectively. These findings indicate that reduction of fragment D led to the formation of NA2, which corresponds to the new right terminal end.

In order to determine the source of fragment NA3, the $400-\mathrm{kb}$ NA3 fragment was recovered with lowmelt agarose and labeled. Hybridization studies of this probe with the PFGE-separated wild-type genomic AseI fragments suggested that either G1 or G2 may be the source of NA3 (Fig. 3B), since G1 and G2 overlap. The NA3 probe also hybridized with fragment $\mathrm{H}$ of SA1-8 and wild-type, because $\mathrm{H}$ was close to NA3, and the recovered NA3 sample used for probe preparation was easily contaminated with DNA from $\mathrm{H}$. Unstable regions are often localized at the telomere or subtelomere of the chromosome in Streptomyces, we therefore firstly attempted to identify the deletion in G2. Southern analysis with probe G2-152 showed that G2 remained intact (Fig. 3B), consistent with PCR results (data not shown). To test the possibility that central fragment G1 underwent deletion to form NA3, we performed hybridization using probe G1-139 located on G1. Probe G1-139 was found to hybridize with NA3 (Fig. 3B), suggesting that NA3 resulted from the reduction of G1.

\section{The extent of deletions and sequence of three junction fragments in SA1-8 chromosome}

To determine the extent of the deletion, we conducted "walking PCR" strategy to detect the relevant region in SA1-8. The entire fragment W and left part of fragment A were missing, and the deletion terminus of fragment A was located near the 691200 nt locus. To confirm the breakpoint, we performed Southern analysis with probe N1 (690197-691592 nt, spanning the $691200 \mathrm{nt}$ locus), which revealed a new 1.84-kb PstI fragment in SA1-8, instead of the 6.4-kb PstI fragment in the wild-type strain (Fig. 4A and 4B). The 1.49-kb fragment was obtained by inverse PCR using primers 113 and 114 (Fig. 4A and 4C). Sequence analysis revealed that the $1.49-\mathrm{kb}$ fragment contained two parts, one from fragment $\mathrm{D}$ in the right chromosomal end, and the other from the remnant of fragment $\mathrm{A}$. The junction sequence was further identified by PCR with primers 118 (located at AseI-D) and 113 (located at AseI-A) (Fig. 4A), using total DNA of SA1-8 as template. The breakpoint of fragment A was determined to be located at 691099 nt, with deletion of the left arm up to 691-kb, and fusion to $8937115 \mathrm{nt}$ on the right chromosomal arm, 88-kb away from the extreme right end (Fig. 4A). Assuming that the 


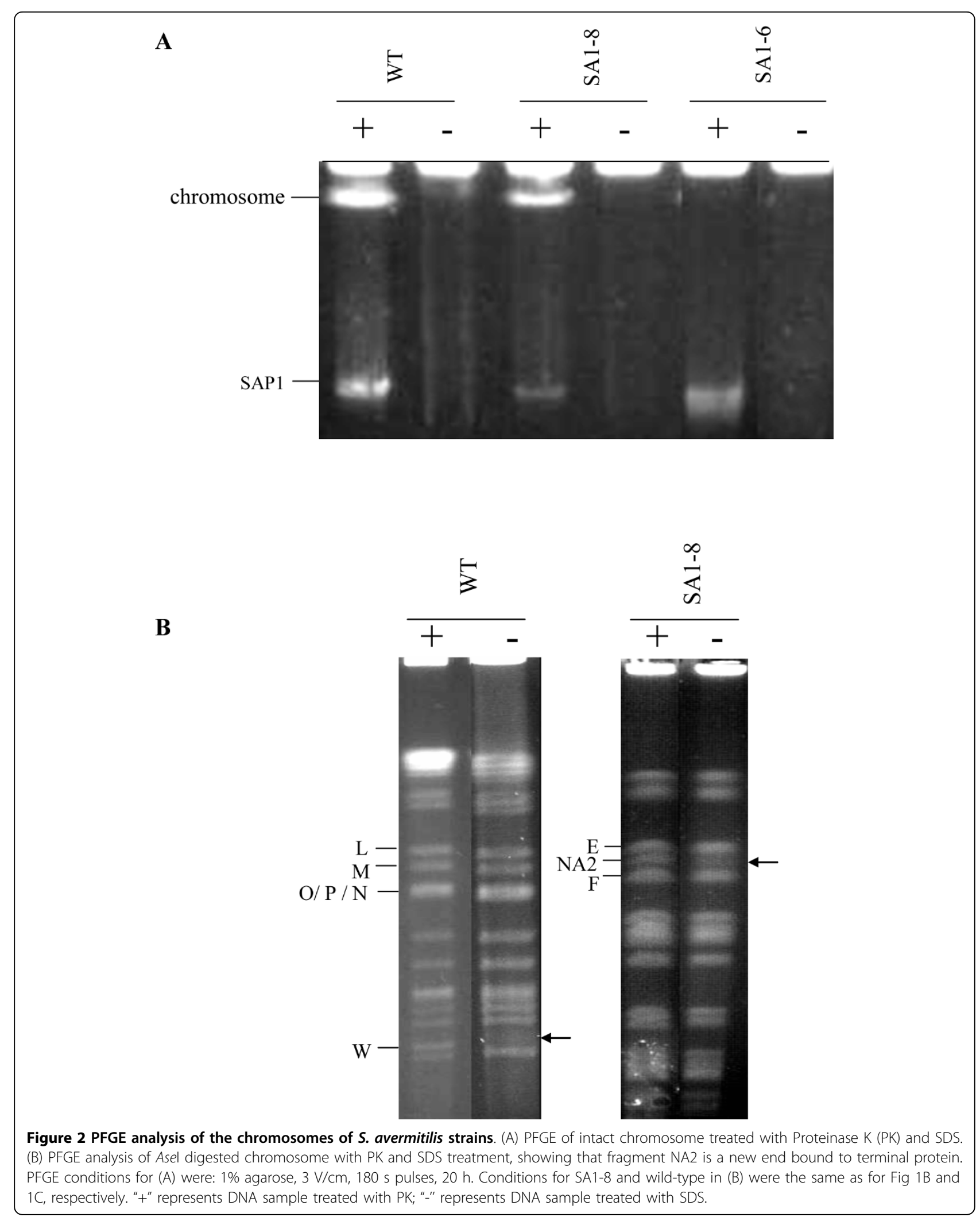




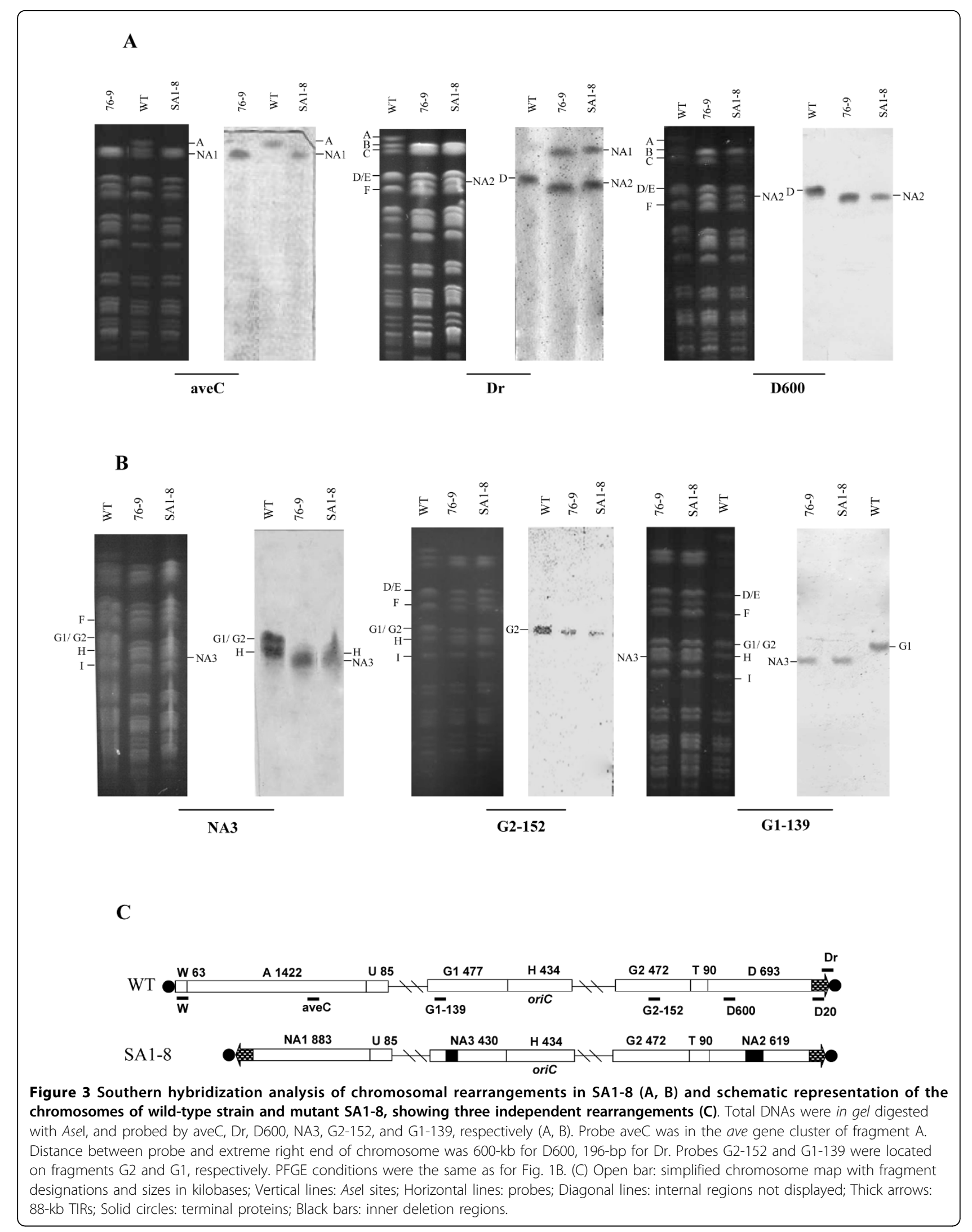


A

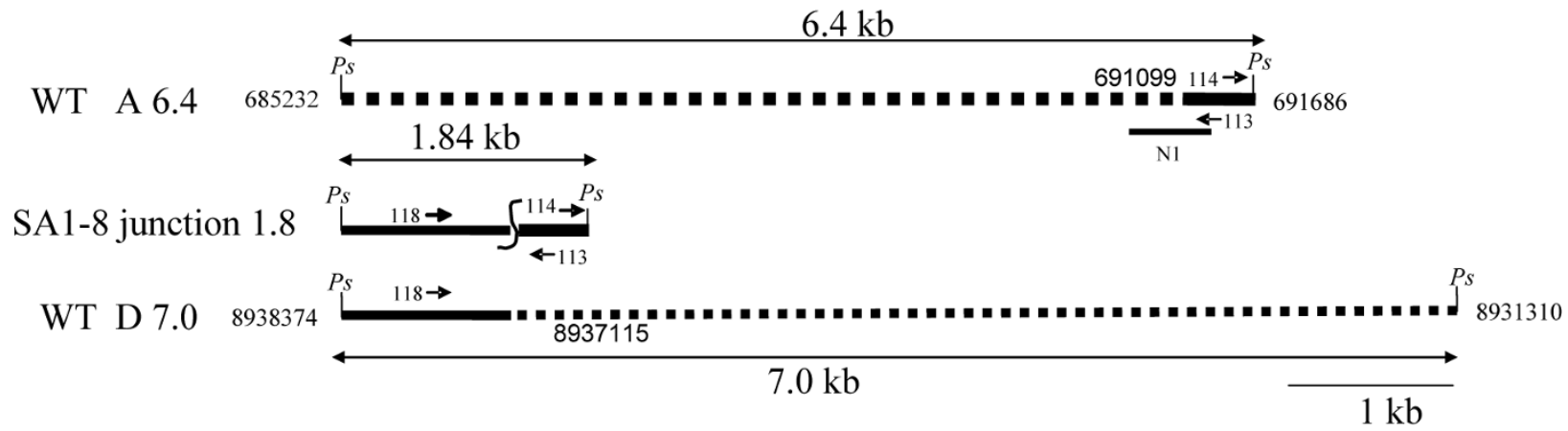

B

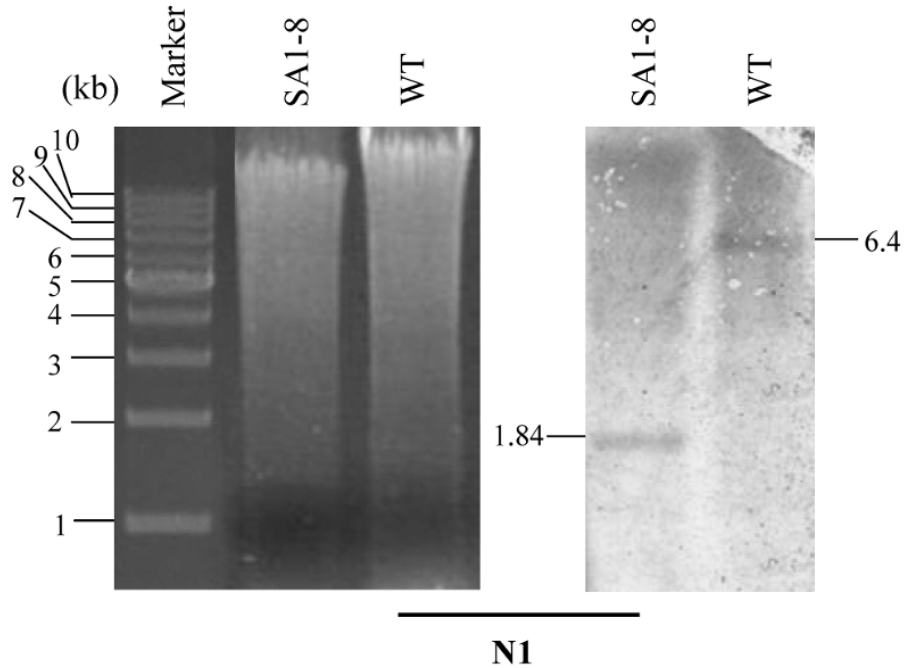

C

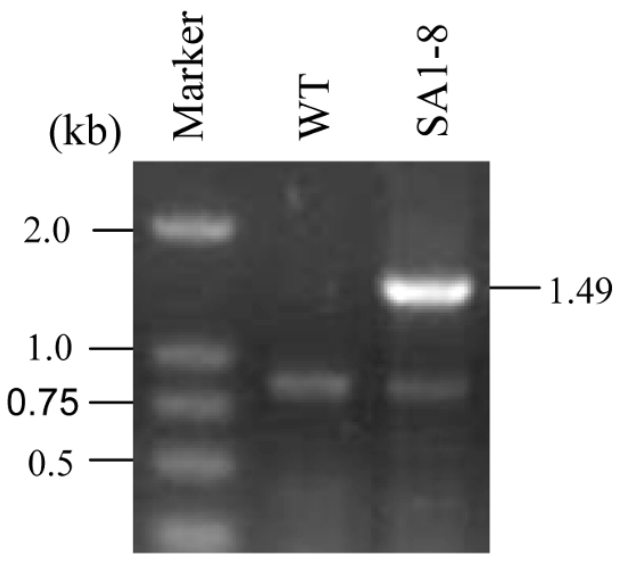

D

WT A 6.4 AGTGTCCGAACACCTTCTTGTTCTTCAGGTTGAAGGTCATCTTCT

SA1-8 J 1.8

WT D 7.0 CGCTTCAGCAGGCCCGGTCTGTTC TTCAGGTTGAAGGTCATCTTCT CGCTTCAGCAGGCCCGGTCTGTTCGCATGGGCGAGCACCAGAAGCC

Figure 4 Analysis of recombination point in fragment NA1. (A) Restriction maps of fragments involved in the recombination event in NA1. The 1.84-kb Pstl junction fragment resulted from fusion in opposite orientation of partially deleted 6.4-kb and 7.0-kb Pstl fragments from left and right chromosomal arms, termed A6.4 and D7.0 respectively. (B) Hybridization analysis of the Pstl fusion fragment. (C) Inverse PCR to obtain the left unknown sequence of 1.84-kb Pstl junction fragment. (D) The fusion sequence in NA1 joins the partial region of fragment A6.4 and D7.0 at a 5-bp overlapping sequence. Bold and non-bold fonts represent nucleotide sequences from fragment A6.4 and D7.0, respectively. Dashed lines represent deleted regions. Ps: Pstl. Primers 113 and 114 were used in inverse PCR. Primers 118 and 113 were used in PCR for amplifying fusion sequence. 
entire right terminal $88-\mathrm{kb}$ end translocated to the left breakpoint to form novel fragment NA1, the size of NA1 was estimated to be $882-\mathrm{kb}(1422 \mathrm{~A}+63 \mathrm{~W}-691+88$ $=882$ ), which is consistent with the finding that NA1 co-migrated with fragment $\mathrm{C}(875-\mathrm{kb})$ in PFGE. This was further confirmed by results from Southern blotting, indicating that NA1 could hybridize with probes D20, D60, and D80 (20-, 60- and 80-kb away from the right extremity, respectively) (data not shown). Comparison of the junction sequence with the right and left sequences from the wild-type strain suggested that a non-homologous recombination event occurred within a short 5-bp region of homology (Fig. 4D).

Walking PCR and sequence analysis showed that the left and right deletion termini in the interior of NA2 were located at $8636494 \mathrm{nt}$ and $8710861 \mathrm{nt}$, respectively (Fig. 5A). The deletion extended to 74-kb, including 64 ORFs (SAV7241-SAV7304). The actual size of NA2 was therefore 619-kb (693D-74 =619). These results also showed that the right terminal 88 -kb fragment was conserved, since the right deletion termini was 314-kb away from the right extremity. We directly amplified and sequenced the newly formed DNA junction sequence with primers 236 and 239 flanking the fusion site. Breakpoint sequence analysis showed that the junction joined the partial regions of left $7.0-\mathrm{kb}$ and right $5.3-\mathrm{kb}$ $K p n \mathrm{I}$ fragments, generating a new KpnI fragment of 8.7kb (Fig. 5A). This was confirmed by hybridization with probe N2 (Fig. 5B). No significant similarity was found when the junction sequence was compared with the left and right sequences from the wild-type strain (Fig. 5C).

The internal deletion region of G1 spanned from $4689788 \mathrm{nt}$ to $4725913 \mathrm{nt}, 562-\mathrm{kb}$ away from the origin of replication (oriC). The results also suggested that the deletion terminated in the left $9.1-\mathrm{kb}$ and right $14.7-\mathrm{kb}$ BamHI fragments, respectively, producing a novel 19.0$\mathrm{kb}$ junction fragment (Fig. 6A). This was confirmed by Southern analysis using probe N3 (Fig. 6B). The fusion sequence acquired by direct PCR amplification with primers 272 and 248 suggested that a non-homologous recombination event had occurred, leading to loss of the intervening 36-kb DNA sequence (Fig. 6C). However, the reduction of $\mathrm{G} 1$ was estimated to be at least $43-\mathrm{kb}$ (477G1-434H $=43$ ), since NA3 was smaller than $\mathrm{H}$ (Fig. 1D). Another small size ( 7-kb) deletion presumably occurred at an undetermined location within G1.

The deleted $36-\mathrm{kb}$ region of G1 contained 32 ORFs from SAV3792 to SAV3823, including 14 hypothetical proteins. Since the substrate mycelia of SA1-8 could form normally, these genes are evidently not essential for growth of $S$. avermitilis. Among these ORFs, 13 genes (40\%) had orthologs in S. coelicolor A3(2), and 12 genes $(37 \%)$ were unique to $S$. avermitilis. The GC content of this region (70.5\%) was not distinct from the average GC content of the $S$. avermitilis chromosome (70.7\%). We did not find any transposable sequences or typical repeated sequences such as tRNA genes flanking the deleted region. It therefore seems unlikely that the deleted region was acquired from other species by horizontal gene transfer.

\section{Similar chromosomal structure of SA1-8 and 76-9}

Based on the results described above, we are able to deduce the chromosomal structure of SA1-8, including at least three independent rearrangements: arm replacement, i.e., the $691-\mathrm{kb}$ left end was deleted, and the 88 -kb right terminal fragment was duplicated and translocated to the left end to form new 88-kb TIRs in SA 18 , in place of the original 174-bp nucleotides in wildtype; the 36-kb deletion within central fragment G1; the 74-kb deletion within right terminal fragment $\mathrm{D}$ (Fig. 3C). Using corresponding primers, the same fusion sequences could be amplified from 76-9 as from SA1-8 (data not shown). Taken together, the PFGE patterns (Fig. 1D) and Southern hybridization results (Fig. 3A and $3 \mathrm{~B}$ ) indicated that 76-9 and SA1-8 have the same chromosomal structure, and have undergone the same three rearrangement events. Since 76-9 is able to sporulate and to produce high-level avermectins, it can be concluded that the deleted central region within G1 is not responsible for the differentiation or avermectin production in S. avermitilis.

\section{Chromosomal circularization in SA1-6}

The 1938-kb deletion region at both chromosomal ends of SA1-6 was identified by walking PCR, including entire Ase I-W, A, U, left part of AseI-P, and right part of AseI-D (Fig. 7A). No obvious retardation of the AseI fragment of SA1-6 was observed in SDS-treated sample (data not shown), together with the intact chromosome remaining trapped in the gel well in PKtreated sample (Fig. 2A), indicating that the SA1-6 chromosome was circularized. The left and right deletion ends were located at $1611078 \mathrm{nt}$ and $8698105 \mathrm{nt}$, respectively. Therefore, the size of the new AseI junction fragment NA4 was $489-\mathrm{kb}$ and overlapped with AseI-G1 in the PFGE gel, which was confirmed by Southern hybridization using probe N4 spanning the fusion site (Additional file 1: Supplementary Fig. S3). Hybridization of probe N4 with the BglII-digested genomic DNA revealed that a 2.99-kb BglII fragment from the left AseI-P and a 13.0-kb BglII fragment from the right AseI-D in the wild-type strain were partially deleted and joined, generating a newly $8.7-\mathrm{kb} B g l \mathrm{II}$ fragment in SA1-6 (Fig. 7B and 7C). No homology was found when the fusion sequence was compared with the corresponding left and right sequences from wildtype (Fig. 7D). 
$\mathbf{A}$

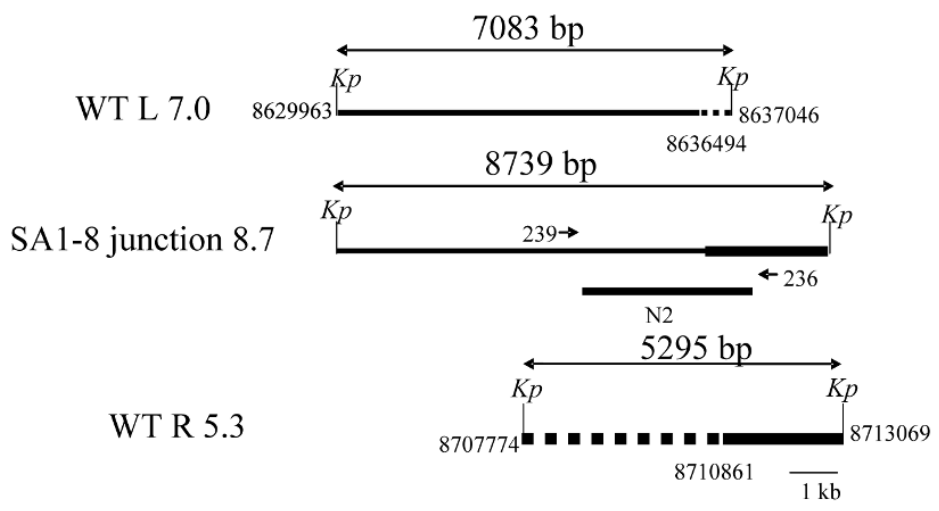

B

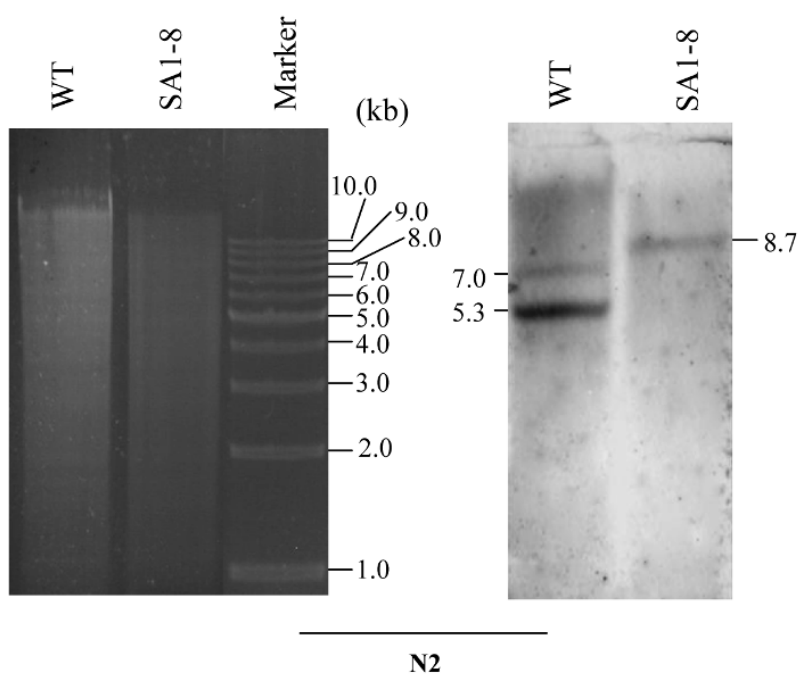

$\mathbf{C}$

WT L 7.0 CGCCGCATCACCGTCGAACTCACCGACGCCGGCCGGGAAGTGAT

\section{SA1-8 J 8.7 CGCCGCATCACCGTCGAACTCACCGGCACTGCACCGACCCCGAC \\ WT R 5.3 GGTCGGCGCCGCGCTGATCGACGCGCACTGCACCGACCCCGAC}

Figure 5 Analysis of fusion sequence in fragment NA2. (A) Location of chromosomal deletion ends and fusion junction. Left and right deletion termini were characterized by stepwise PCR mapping. Deleted and fused regions are indicated by dashed and shaded lines, respectively. Kp, Kpnl. (B) Southern analysis of fusion fragment with probe N2, which was prepared using primers 236 and 239 . (C) Junction sequence, showing no obvious homology between the original sequences.

Stability assay of chromosomal structure in bald mutants Generational studies were performed to assess the chromosomal stability of bald mutants derived from the wild-type strain. Four bald strains were selected, and subjected to PFGE analysis following ten passages. The chromosomal structure of SA1-8 and SA1-6 was conserved, whereas that of SA1-7 and SA3-1 was changed (Additional file 1: Supplementary Fig. S4A). Both SA1-7 and SA3-1 lost their characteristic bands, and became indistinguishable from SA1-6. SA1-7 chromosome was further monitored in each passage, and found to change in the 4th passage (Additional file 1: Supplementary Fig. S4B). The corresponding fusion fragments of SA1-6 and SA1-8 were also detected in their progeny. These results indicate that chromosomal structure of SA1-6 and SA18 is stable. 


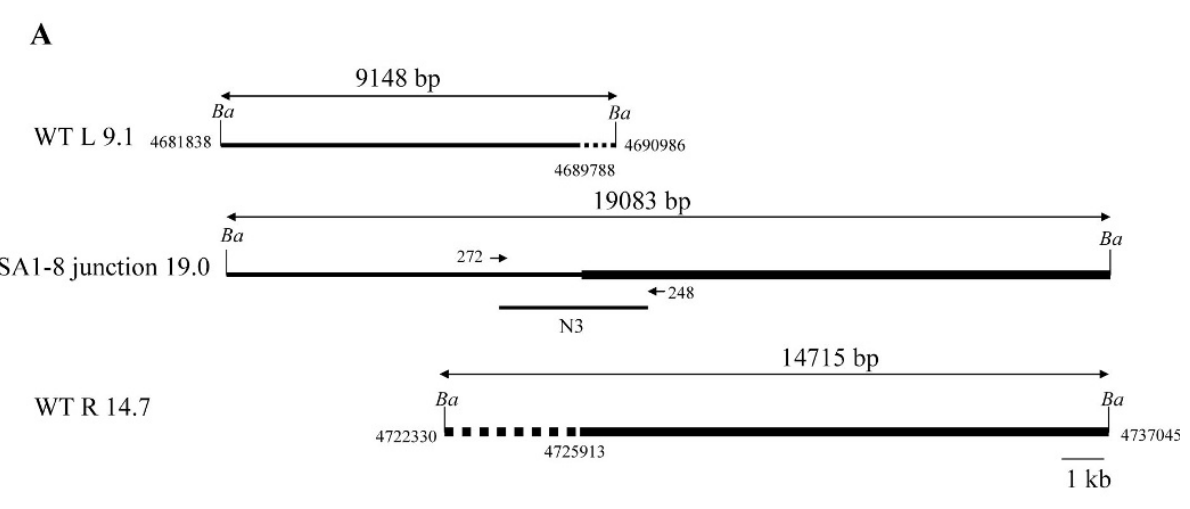

B

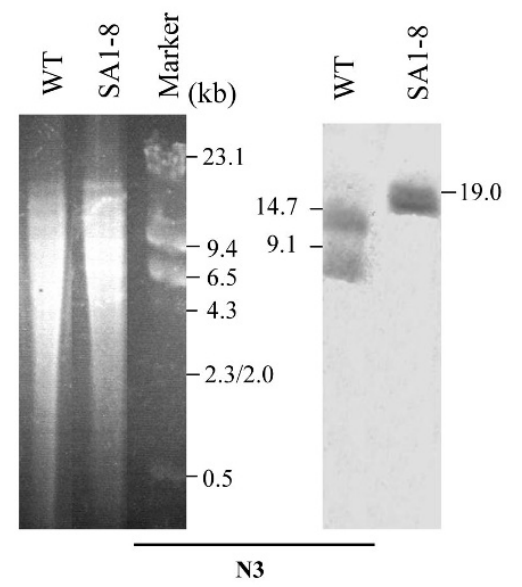

C

WT L 9.1 GAATGGTGACGGCCCCGCCGGCCTGCTGGGCGCGGAC

SA1-8 J 19.0 GAATGGTGACGGCCCCGCCGCTCTTGCGCAGGCCGACC

WT R 14.7 CGTTGACGCACCGGGTCCCGCTCTTGCGCAGGCCGACC

Figure 6 Analysis of fusion sequence in fragment NA3. (A) Location of chromosomal deletion ends and fusion junction. Ba, BamHI. (B) Southern analysis of junction fragment with probe N3, which was prepared using primers 248 and 272. (C) Junction sequence in NA3. The 3-bp overlapping sequence is boxed.

\section{Discussion}

This study demonstrated for the first time chromosomal rearrangement events in $S$. avermitilis, including chromosomal arm replacement, internal deletions and circularization. The chromosomal arm replacement in the bald mutant SA1-8 consisted of deletion of the 691-kb left terminus, and duplication of the $88-\mathrm{kb}$ right terminus. The resulting new junction in fragment NA1 joined the partial coding regions of SAV546 (putative dehydrogenase) and SAV7499 (putative two-component system response regulator) at a 5 -bp overlapping sequence. The internal deletions of fragments D and G1 appeared to be direct recombination events between two points. Fragment D was reduced 74-kb from SAV7241 to
SAV7304. No significant homology was found, since the former was a putative ATP-dependent Clp protease, and the latter was a hypothetical protein. G1 had a 36-kb deletion, from SAV3792 to SAV3823, and the left and right deletion termini overlapped only by 3 -bp nucleotides. The circular chromosome of SA1-6 joined SAV1302 (acetyl xylan esterase) and SAV7294 (amino acid transporter protein) with no overlapping sequence. Thus, all fusion sequences displayed minimal or no homology, indicating that the chromosome alteration has resulted from non-homologous recombination. Similarly, non-homologous (sometimes termed "illegitimate") recombination appeared to be involved in nearly all rearrangement events in previous studies of genetic 


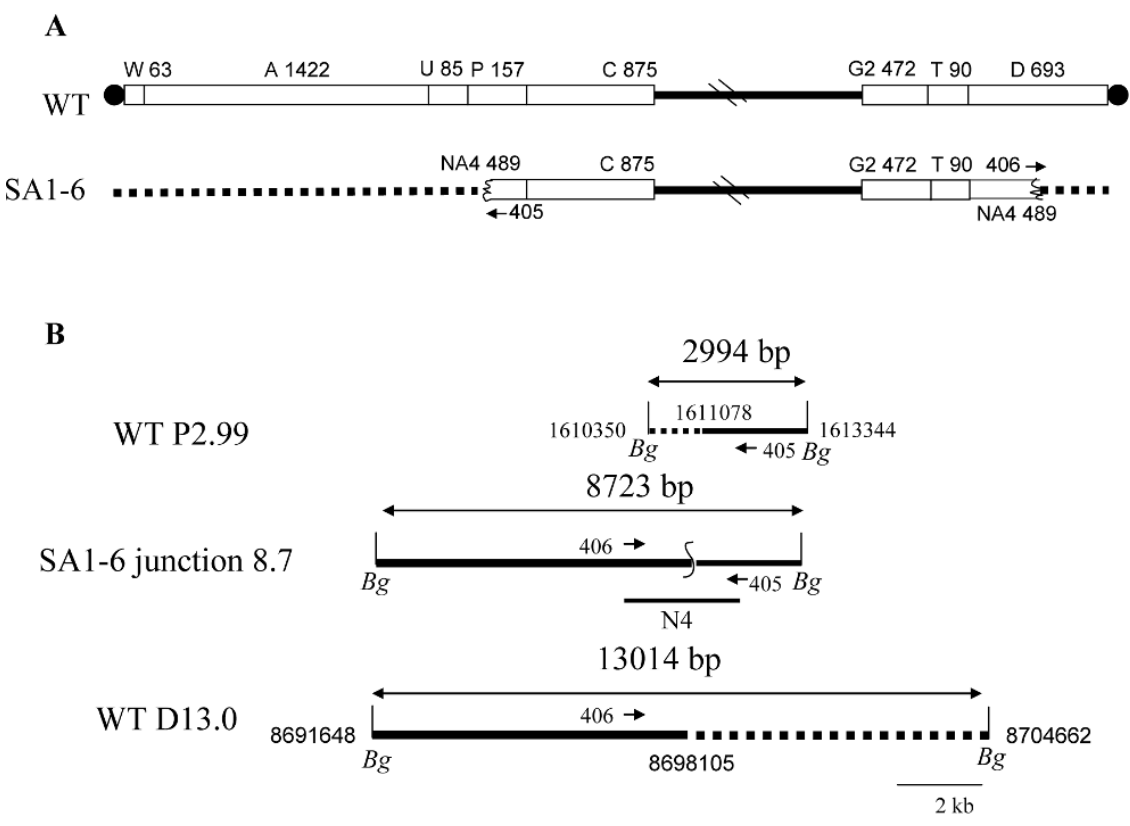

C

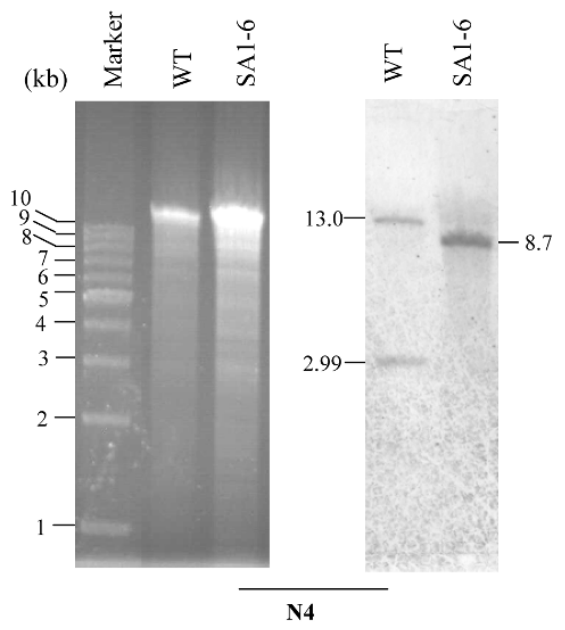

D

WT P 2.99 ATGCCGCCGCCCTGGCTGGTGCCGACGGCCGCGACGCGGGCGGA SA1-6 J 8.7 ATGCCGCCGCCCTGGCTGGTGCCGACATCTTCTCGATCTGGCTGAT

WT D13.0 GATGCCGTACGTCCCGGCGCTGGGGGTGATCTTCTCGATCTGGCTGAT

Figure 7 Characterization of circular chromosome in SA1-6. (A) Schematic representation of the chromosomes of wild-type strain and mutant SA1-6, showing deletions at both ends. (B) Location of chromosomal deletion ends and fusion junction. Bg, Bg/ll. (C) Southern analysis of fusion fragment with probe N4, which was prepared using primers 405 and 406 . (D) Junction sequence, showing no obvious homology between the original sequences.

instability in other Streptomyces species [5,9,12,14,21,25], except for two homologous recombinations occurring between duplicated genes $[8,11]$. This is reminiscent of breakpoint analysis of genome rearrangements in Saccharomyces cerevisiae, in which non-homologous endjoining (NHEJ) appeared to be the major mechanism involved in gross chromosomal rearrangements, even in those strains in which homologous recombination is functional [26]. Homologs of the eukaryotic DNA-endbinding repair protein $\mathrm{Ku}$, involved in NHEJ pathway, have been found in Streptomyces [27], suggesting the presence of this pathway. It would thus be of interest to 
determine the relationship between $\mathrm{Ku}$ protein and chromosome instability in $k u$ mutants of Streptomyces.

This is the first report of an inner deletion event involving the central region of the Streptomyces chromosome, suggesting that each part of the Streptomyces chromosome may be the target for rearrangements. Previous reports indicated that the two chromosome ends were primary targets for a variety of rearrangements: deletion, amplification, replacement, and circularization $[5,9,14,25]$. No essential genes located in the telomeric or subtelomeric regions of Streptomyces chromosome, and we are able to observe and characterize only those rearrangement events which did not affect the growthdependent genes. This is the most likely reason as to why the majority of the rearrangements described in previous studies are located in the chromosome arms. The deleted central regions revealed in the present study are located between chromosomal region B (position 4,313,571-4,591,925 nt, SAV3480-SAV3709) and oriC (position 5,287,935- 5,289,024 nt) [20]. The 32 missing ORFs (Additional file 2) are unlikely to include any putative essential genes, since mutants SA1-8 and 76-9 both grew well on solid or in liquid medium. Similarly, Putnam et al. observed that any chromosomal region except centromeres in S. cerevisiae could be targeted by genome rearrangement, based on distribution of rearrangements in non-repetitive regions of the genome [26].

We found that the chromosomal structures of mutants SA1-8 and 76-9 were quite similar. The former resulted from spontaneous mutation of the wild-type strain, and the latter from various mutagenic treatments (UV, NTG, etc.). The phenotypes of SA1-8 and 76-9 were obviously distinct: SA1-8 was bald and did not produce avermectins, whereas 76-9 produced high level of avermectins and developed rich spores. Such differences presumably resulted from point mutations or small fragment changes involved in avermectin production and differentiation. On the other hand, some normal gray colonies of 76-9 underwent sequential differentiation into bald colonies, which remained the same chromosomal framework. This suggested that a chromosomal structure like that of 76-9 was relative stable. From a practical point of view, it would be valuable to complement such bald mutants with a gene library from 76-9 or the wild-type strain. If some mutation hot spots were identified and suppressed artificially, it would be possible to construct stable, high avermectin-producing strains. Such possibilities are being currently considered as part of ongoing studies in our laboratory.

Previous studies showed that artificially or naturally circularized chromosome of Streptomyces usually exhibited genetic instability similar to or at higher rates than the parent linear chromosome $[7,17,18]$. One possible explanation for the instability of circular chromosomes is lack of replication terminator structures or segregation elements, which are both necessary to maintain chromosome integrity [7]. However, two mutants, 40423 and N2 from S. griseus, stably maintained their circular chromosomes [9], as was the case for mutant SA1-6 in the present work. It was postulated by Kameoka et al. that circularization prevented deletions from progressing into indispensable regions [9]. However, the regions near the deletion ends in SA1-6 don't contain any essential genes and thus the cause for stability of circular chromosomes in Streptomyces still remains to be elucidated.

Notably, we found that the essential chromosome structures of genetic instability mutants SA1-8 and SA16 were retained, whereas other dynamic mutants such as SA1-7 and SA3-1 underwent continuous chromosomal rearrangement. Similar phenomena were observed in S. coelicolor [14]. The mechanisms driving such gradual alterations of chromosomes are unclear. Alteration of an unstable monocentric chromosome in S. cerevisiae was attributed to joining of two "incompatible" regions [28]. In analogy, a plausible hypothesis in the present study is that the chromosomes of $S$. avermitilis mutants SA1-8 and SA1-6 were formed compatibly, whereas chromosomes of SA1-7 and SA3-1 harbored incompatible junction. However, what makes a stable junction "compatible", and what leads to "incompatibility" of two chromosome regions, remain to be clarified. Breakpoint analysis of the unstable chromosome of SA1-7 may shed some light on this issue.

The inherent chromosome instability of Streptomyces likely reflects an evolutionary strategy for adapting to environmental changes by creating populations with altered genetic information [29]. Unfortunately, this "strategy" often results in reduced production of secondary metabolites which are desired in agricultural, pharmaceutical, and research industries. From this point of view, the present findings contribute to elucidation of mechanisms underlying genetic instability in Streptomyces, and may help devising approaches to suppress or control such instability for industrial purposes.

\section{Conclusions}

S. avermitilis underwent chromosomal rearrangement events, including chromosomal arm replacement, internal deletions and circulation, by non-homologous recombination. The fact that major deletion in the central region of chromosome was observed in S. avermitilis suggests that genetic instability of the Streptomyces chromosome is uniform across the entire chromosome. Stability assay showed that the chromosome of some bald mutants derived from the wild-type strain was 
conserved, whereas other mutants underwent further chromosomal rearrangement.

\section{Methods}

Bacterial strains and growth conditions

S. avermitilis ATCC31267 (wild-type strain) was used as starting strain and control. 76-9 was a high avermectinproducing strain derived from ATCC31267 by continuous mutagenesis, with the ability to sporulate. Spontaneous "bald" mutants (i.e., defective in production of aerial mycelia) of ATCC31267 and 76-9 were picked at random for further study, since the bald phenotype was stable. All strains were grown at $28^{\circ} \mathrm{C}$ on YMS solid medium for sporulation [30], or for isolation and growth of bald colonies.

\section{Preparation of DNA for PFGE analysis}

S. avermitilis was cultured at $28^{\circ} \mathrm{C}$ for $36 \mathrm{~h}$ in $25 \mathrm{~mL}$ YEME with $25 \%$ sucrose in a $250 \mathrm{~mL}$ flask, containing a coiled stainless steel spring to promote aeration and cell dispersion. Mycelia were harvested and used for making plugs, as described by Kieser et al [31]. For restriction analysis, $200 \mu \mathrm{l}$ buffer (per manufacturer's instructions) was added into $1.5 \mathrm{~mL}$ eppendorf tube containing one plug, incubated for $30 \mathrm{~min}$ at room temperature, and then the buffer was replaced with $300 \mu \mathrm{l}$ fresh buffer containing $2 \mu \mathrm{l} \mathrm{BSA}(100 \mu \mathrm{g} / \mathrm{mL})$ and $50 \mathrm{U}$ AseI to digest the plug for $4 \mathrm{~h}$ at $37^{\circ} \mathrm{C}$. PFGE runs were performed in a CHEF MAPPER XA system (Bio-Rad). Agarose gels were run in $0.5 \times \mathrm{TBE}$ buffer at $14^{\circ} \mathrm{C}$. Pulse times were optimized depending on the sizes of the DNA fragments to be separated. Proteinase $\mathrm{K}$ or SDS-treated DNA samples were prepared as described by Kieser et al [31].

Detection of chromosomal deletion by PCR amplification Using the available genomic sequence of $S$. avermitilis http://avermitilis.ls.kitasato-u.ac.jp, PCR primers were designed to detect chromosomal deletion, with DNA extracted from the wild-type strain as positive control.

\section{Inverse PCR}

To determine the fusion sequence in novel fragment AseI-NA1 of mutant strain SA1-8, inverse PCR was conducted. Total DNA of strain SA1-8 was completely digested by PstI, and separated by conventional agarose

Table 1 Primers used for preparation of probes

\begin{tabular}{|c|c|c|c|}
\hline Probes & & Primers sequence $\left(5^{\prime}-3^{\prime}\right)$ & Description \\
\hline \multirow[t]{2}{*}{ W } & 11 & GTTGCGGACGTGTGACTTG & to detect the left extremity of chromosome \\
\hline & 12 & GAACTACATGCCGGGAGTG & \\
\hline \multirow[t]{2}{*}{ aveC } & 15 & CAGCAAGGATACGGGGAC & to detect the avermectin biosynthetic gene cluster \\
\hline & 16 & ACCGAGCACGATGCCGATG & \\
\hline \multirow[t]{2}{*}{ G1-139 } & 139 & GGTTGACGACGTCCTTGAG & to detect fragment Asel-G1 \\
\hline & 140 & CGACACTCATGAAGCGACC & \\
\hline \multirow[t]{2}{*}{ G2-152 } & 152 & CTGCTCAAGACGAAGGTGC & to detect fragment Asel-G2 \\
\hline & 153 & CCGTCACATCGCTGTCATG & \\
\hline \multirow[t]{2}{*}{ D600 } & 131 & GTGATCGTGAAGACCTCGC & to detect fragment Asel-D \\
\hline & 132 & CTCCACCATGACAAGACCG & \\
\hline \multirow[t]{2}{*}{$\operatorname{Dr}$} & 19 & AGTCGTACGTCCGCAACTG & to detect the right extremity of chromosome \\
\hline & 20 & AGGTCTTCCGCTTCGCTTC & \\
\hline \multirow[t]{2}{*}{ D20 } & 125 & CTCTAGACGGCGGAATCAC & to detect the duplication and translocation of right $88-\mathrm{kb}$ end \\
\hline & 126 & GCGACAAGGGCTAAGACTC & \\
\hline \multirow[t]{2}{*}{ D60 } & 96 & GTTCTGGCAGTCGTCGTAG & to detect the duplication and translocation of right $88-\mathrm{kb}$ end \\
\hline & 97 & TGAAGAAGACCCGGTCTGG & \\
\hline \multirow[t]{2}{*}{ D80 } & 98 & ACGAACGTGCCCTGCTCAC & to detect the duplication and translocation of right $88-\mathrm{kb}$ end \\
\hline & 99 & GGTGACGAGTTCGGAGACG & \\
\hline \multirow[t]{2}{*}{ N1 } & 75 & GGAGGTAGCGGATGTTGTG & to detect the rearrangement event in fragment Asel-NA1 \\
\hline & 76 & CTGGTCCCAGTACACGATC & \\
\hline \multirow[t]{2}{*}{ N2 } & 236 & GGCTCGTTCATCTTCCTCG & to detect the rearrangement event in fragment Asel-NA2 \\
\hline & 239 & GCACATCAGAGGGTCATGC & \\
\hline \multirow[t]{2}{*}{ N3 } & 272 & CGTTGACGTAGAGCTGCG & to detect the rearrangement event in fragment Asel-NA3 \\
\hline & 248 & ACCTGAGCAGCTCGTGAAG & \\
\hline \multirow[t]{2}{*}{ N4 } & 405 & TGTGACGGTGTGCCAGTAG & to detect the rearrangement event in fragment Asel-NA4 \\
\hline & 406 & ATGCCCTCGACTACGACAC & \\
\hline
\end{tabular}


gel electrophoresis. Fragments from 1-3 kb were retrieved, purified, and self-ligated. Circular DNA was used as template for inverse PCR. Since walking PCR and Southern blotting analysis had revealed the formation of a new $\sim 2 \mathrm{~kb}$ PstI fragment spanning the breakpoint of NA1 and the sequence of the right part of this new Pst fragment (see the results), two primers, 113 (GGACTACGCCTTCGACTTC) and 114 (GATCGTGTACTGGGACCAG) in the right known region of the new PstI fragment, were designed in opposite directions to amplify the left unknown sequence. Primer 113 was close to the breakpoint and primer 114 was near the right PstI site. After sequencing analysis of the inverse PCR product, primers 118 (GTATCTCTCGTACGCCTCG) and 113 were used to determine the junction sequence (Fig. 4A).

\section{DNA labeling and hybridization}

Following PFGE or conventional agarose gel electrophoresis, DNA fragments were transferred to nylon filters (Hybond-N, Millipore) by capillary method [32], and cross-linked by exposure to $254 \mathrm{~nm}$ UV for $10 \mathrm{~min}$. DNA probes for Southern blotting were amplified by PCR, and labeled using a nonradioactive digoxigenin (DIG) labeling kit (Roche). Hybridization and detection were performed according to the manufacturer's instructions. Primers used for preparation of probes are listed in Table 1.

\section{Chromosomal stability assay of mutants}

To assess chromosomal stability of mutants over generations, selected bald mutants derived from wild-type were grown for ten passages on YMS plates. Since aerial mycelia of $S$. avermitilis begin to emerge after $48 \mathrm{~h}$ of incubation on YMS, we transferred mycelia of bald mutants grown for 3 days by streaking on YMS plates. Genomic DNA was analyzed by PFGE as described above.

\section{Additional material}

Additional file 1: Supplementary Fig. S1. Asel restriction patterns of genomic DNA of spontaneous bald mutants from 76-9. Supplementary Fig. S2. Southern hybridization analysis of the left (A) and right end (B) of the SA1-8 chromosome. Supplementary Fig. S3. Southern

hybridization analysis of Asel macrorestriction fragments of the SA1-6 chromosome with probe N4. Supplementary Fig. S4. Generational stability analysis of bald mutants.

Additional file 2: Complete data for deletion extent of fragment G1.

\section{Acknowledgements}

This work was supported by grants from the National Natural Science Foundation of China (Grant No. 30670037) and the National Basic Research Program of China (Grant No. 2009CB118905).

\section{Authors' contributions}

WC carried out most of the experiments and wrote the draft manuscript. FH and XZ performed some research on characterizing the circular chromosome of mutant SA1-6. ZC assisted with experimental design and data analysis. YW and $J L$ supervised the whole work and revised the manuscript. All authors read and approved the final manuscript.

Received: 19 April 2010 Accepted: 26 July 2010 Published: 26 July 2010

\section{References}

1. Demain AL: Pharmaceutically active secondary metabolites of microorganisms. Appl Microbiol Biotechnol 1999, 52(4):455-463.

2. Bentley SD, Chater KF, Cerdeno-Tarraga AM, Challis GL, Thomson NR, James KD, Harris DE, Quail MA, Kieser H, Harper D, Bateman A, Brown S, Chandra G, Chen CW, Collins M, Cronin A, Fraser A, Goble A, Hidalgo J, Hornsby T, Howarth S, Huang CH, Kieser T, Larke L, Murphy L, Oliver K, O'Neil S, Rabbinowitsch E, Rajandream MA, Rutherford K, Rutter S, Seeger K, Saunders D, Sharp S, Squares R, Squares S, Taylor K, Warren T, Wietzorrek A, Woodward J, Barrell BG, Parkhill J, Hopwood DA: Complete genome sequence of the model actinomycete Streptomyces coelicolor A3(2). Nature 2002, 417(6885):141-147.

3. Lin YS, Kieser HM, Hopwood DA, Chen CW: The chromosomal DNA of Streptomyces lividans 66 is linear. Mol Microbiol 1993, 10(5):923-933.

4. Omura S, Ikeda H, Ishikawa J, Hanamoto A, Takahashi C, Shinose M, Takahashi Y, Horikawa H, Nakazawa H, Osonoe T, Kikuchi H, Shiba T, Sakaki Y, Hattori M: Genome sequence of an industrial microorganism Streptomyces avermitilis: deducing the ability of producing secondary metabolites. Proc Natl Acad Sci USA 2001, 98(21):12215-12220.

5. Volff JN, Altenbuchner J: Genetic instability of the Streptomyces chromosome. Mol Microbiol 1998, 27(2):239-246.

6. Volff JN, Vandewiele D, Decaris B: Stimulation of genetic instability and associated large genomic rearrangements in Streptomyces ambofaciens by three fluoroquinolones. Antimicrob Agents Chemother 1994, 38(9):1984-1990.

7. Fischer G, Decaris B, Leblond P: Occurrence of deletions, associated with genetic instability in Streptomyces ambofaciens, is independent of the linearity of the chromosomal DNA. J Bacterio/ 1997, 179(14):4553-4558,

8. Fischer $G$, Wenner $T$, Decaris $B$, Leblond P: Chromosomal arm replacement generates a high level of intraspecific polymorphism in the terminal inverted repeats of the linear chromosomal DNA of Streptomyces ambofaciens. Proc Natl Acad Sci USA 1998, 95(24):14296-14301.

9. Kameoka D, Lezhava A, Zenitani H, Hiratsu K, Kawamoto M, Goshi K, Inada K, Shinkawa H, Kinashi H: Analysis of fusion junctions of circularized chromosomes in Streptomyces griseus. J Bacteriol 1999, 181(18):5711-5717.

10. Redenbach M, Flett F, Piendl W, Glocker I, Rauland U, Wafzig O, Kliem R, Leblond P, Cullum J: The Streptomyces lividans 66 chromosome contains a $1 \mathrm{MB}$ deletogenic region flanked by two amplifiable regions. Mol Gen Genet 1993, 241(3-4):255-262.

11. Uchida T, Miyawaki M, Kinashi H: Chromosomal arm replacement in Streptomyces griseus. J Bacteriol 2003, 185(3):1120-1124.

12. Wenner T, Roth V, Fischer G, Fourrier C, Aigle B, Decaris B, Leblond P: Endto-end fusion of linear deleted chromosomes initiates a cycle of genome instability in Streptomyces ambofaciens. Mol Microbiol 2003, 50(2):411-425.

13. Widenbrant EM, Tsai HH, Chen CW, Kao CM: Spontaneous amplification of the actinorhodin gene cluster in Streptomyces coelicolor involving native insertion sequence IS466. J Bacterio/ 2008, 190(13):4754-4758.

14. Widenbrant EM, Tsai HH, Chen CW, Kao CM: Streptomyces coelicolor undergoes spontaneous chromosomal end replacement. J Bacteriol 2007, 189(24):9117-9121.

15. Yanai $K$, Murakami T, Bibb M: Amplification of the entire kanamycin biosynthetic gene cluster during empirical strain improvement of Streptomyces kanamyceticus. Proc Natl Acad Sci USA 2006, 103(25):9661-9666.

16. Yu TW, Chen CW: The unstable melC operon of Streptomyces antibioticus is codeleted with a Tn4811-homologous locus. J Bacteriol 1993, 175(6):1847-1852.

17. Lin YS, Chen CW: Instability of artificially circularized chromosomes of Streptomyces lividans. Mol Microbiol 1997, 26(4):709-719.

18. Volff JN, Viell P, Altenbuchner J: Artificial circularization of the chromosome with concomitant deletion of its terminal inverted repeats 
enhances genetic instability and genome rearrangement in Streptomyces lividans. Mol Gen Genet 1997, 253(6):753-760.

19. Burg RW, Miller BM, Baker EE, Birnbaum J, Currie SA, Hartman R, Kong YL, Monaghan RL, Olson G, Putter I, Tunac JB, Wallick H, Stapley EO, Oiwa R, Omura S: Avermectins, new family of potent anthelmintic agents: producing organism and fermentation. Antimicrob Agents Chemother 1979, 15(3):361-367.

20. Ikeda H, Ishikawa J, Hanamoto A, Shinose M, Kikuchi H, Shiba T, Sakaki Y, Hattori M, Omura S: Complete genome sequence and comparative analysis of the industrial microorganism Streptomyces avermitilis. Nat Biotechnol 2003, 21(5):526-531.

21. Birch A, Hausler A, Ruttener C, Hutter R: Chromosomal deletion and rearrangement in Streptomyces glaucescens. J Bacteriol 1991, 173(11):3531-3538

22. Gravius B, Bezmalinovic T, Hranueli D, Cullum J: Genetic instability and strain degeneration in Streptomyces rimosus. Appl Environ Microbiol 1993, 59(7):2220-2228.

23. Leblond $P$, Demuyter $P$, Simonet $J M$, Decaris B: Genetic instability and associated genome plasticity in Streptomyces ambofaciens: pulsed-field gel electrophoresis evidence for large DNA alterations in a limited genomic region. J Bacteriol 1991, 173(13):4229-4233.

24. Leblond $P$, Demuyter $P$, Simonet JM, Decaris B: Genetic instability and hypervariability in Streptomyces ambofaciens: towards an understanding of a mechanism of genome plasticity. Mol Microbiol 1990, 4(5):707-714.

25. Leblond P, Decaris B: New insights into the genetic instability of Streptomyces. FEMS Microbiol Lett 1994, 123(3):225-232.

26. Putnam CD, Pennaneach V, Kolodner RD: Saccharomyces cerevisiae as a model system to define the chromosomal instability phenotype. Mol Cell Biol 2005, 25(16):7226-7238.

27. Aravind $\mathrm{L}$, Koonin EV: Prokaryotic homologs of the eukaryotic DNA-endbinding protein $\mathrm{Ku}$, novel domains in the Ku protein and prediction of a prokaryotic double-strand break repair system. Genome Res 2001, 11(8):1365-1374

28. Admire A, Shanks L, Danzl N, Wang M, Weier U, Stevens W, Hunt E, Weinert T: Cycles of chromosome instability are associated with a fragile site and are increased by defects in DNA replication and checkpoint controls in yeast. Genes \& Dev 2006, 20(2):159-173.

29. Chen CW, Huang CH, Lee HH, Tsai HH, Kirby R: Once the circle has been broken: dynamics and evolution of Streptomyces chromosomes. Trends Genet 2002, 18(10):522-529.

30. Ikeda H, Kotaki H, Tanaka H, Ōmura S: Involvement of glucose catabolism in avermectin production by Streptomyces avermitilis. Antimicrob Agents Chemother 1988, 32(2):282-284.

31. Kieser T, Bibb MJ, Buttner MJ, Chater KF, Hopwood DA: Practical Streptomyces Genetics. Norwich: John Innes Foundation 2000.

32. Smith GE, Summers MD: The bidirectional transfer of DNA and RNA to nitrocellulose or diazobenzyloxymethyl-paper. Anal Biochem 1980, 109(1):123-129.

doi:10.1186/1471-2180-10-198

Cite this article as: Chen et al: Chromosomal instability in Streptomyces avermitilis: major deletion in the central region and stable circularized chromosome. BMC Microbiology 2010 10:198.

\section{Submit your next manuscript to BioMed Central and take full advantage of:}

- Convenient online submission

- Thorough peer review

- No space constraints or color figure charges

- Immediate publication on acceptance

- Inclusion in PubMed, CAS, Scopus and Google Scholar

- Research which is freely available for redistribution

Submit your manuscript at www.biomedcentral.com/submit
Biomed Central 